\title{
BEATBOX v1.0: Background Error Analysis Testbed with Box Models
}

\author{
Christoph Knote ${ }^{1}$, Jérôme Barré ${ }^{2}$, and Max Eckl ${ }^{1, a}$ \\ ${ }^{1}$ Meteorological Institute, LMU, 80333 Munich, Germany \\ ${ }^{2}$ European Centre for Medium-Range Weather Forecasts, Shinfield Park, Reading, RG2 9AX, UK \\ anow at: Institute of Atmospheric Physics, DLR, 82234 Oberpfaffenhofen, Germany
}

Correspondence: Christoph Knote (christoph.knote@physik.uni-muenchen.de) and Jérôme Barré (jerome.barre@ecmwf.int)

Received: 12 August 2017 - Discussion started: 4 October 2017

Revised: 19 December 2017 - Accepted: 2 January 2018 - Published: 8 February 2018

\begin{abstract}
The Background Error Analysis Testbed (BEAT$\mathrm{BOX})$ is a new data assimilation framework for box models. Based on the BOX Model eXtension (BOXMOX) to the Kinetic Pre-Processor (KPP), this framework allows users to conduct performance evaluations of data assimilation experiments, sensitivity analyses, and detailed chemical scheme diagnostics from an observation simulation system experiment (OSSE) point of view. The BEATBOX framework incorporates an observation simulator and a data assimilation system with the possibility of choosing ensemble, adjoint, or combined sensitivities. A user-friendly, Python-based interface allows for the tuning of many parameters for atmospheric chemistry and data assimilation research as well as for educational purposes, for example observation error, model covariances, ensemble size, perturbation distribution in the initial conditions, and so on. In this work, the testbed is described and two case studies are presented to illustrate the design of a typical OSSE experiment, data assimilation experiments, a sensitivity analysis, and a method for diagnosing model errors. BEATBOX is released as an open source tool for the atmospheric chemistry and data assimilation communities.
\end{abstract}

\section{Introduction}

Current regional and global models of the composition of Earth's atmosphere exhibit a high level of complexity due to the combination of chemical and meteorological processes such as transport, thermodynamics, radiation, or precipitation. But "just" gas-phase chemistry itself already has a considerable amount of complexity. The number of variables (chemical compounds) and equations (chemical reactions) in current model representations of tropospheric chemistry ("mechanisms") can vary by 2 orders of magnitude (from $10^{2}$ to $10^{4}$ ). Compare, for example, two well-known mechanisms: the Model for Ozone And Related Chemical Tracers version T1 (MOZART-T1; Emmons et al., 2010; Knote et al., 2014) uses 134 species and 250 reactions, whereas the Master Chemical Mechanism version 3.3 (MCMv3.3; Jenkin et al., 2015) employs over 5000 species and over 15000 reactions. MCMv3.3 is called a "near-explicit" chemical mechanism, which describes in detail the gas-phase chemical processes involved in the tropospheric degradation of volatile organic compounds (VOCs). On the other hand, MOZARTT1 uses a simplified ("lumped") representation of VOCs. This reduction in complexity is advantageous as it reduces the computational demand, but can lead to significant errors in the prediction of atmospheric composition. The choice of a chemical mechanism is therefore a trade-off: while MCMv3.3 would be desirable due to its fidelity in representing atmospheric chemistry, it cannot currently be used in large-scale 3-D atmospheric simulation due to its computational demand. MOZART-T1 is less accurate, but also much more economic.

Investigating the performance of chemical mechanisms is often done using zero-dimensional (0-D) box models. Numerous studies have used box models to study reactive gasphase chemistry and provide intercomparisons and validation of mechanisms. Emmerson et al. (2009) provided a comprehensive comparison of the MCM mechanism with six tropospheric chemistry lumped schemes that could be used within chemistry transport models. Archibald et al. (2010) 
performed an intercomparison of the gas-phase mechanism for isoprene degradation in a box model with various mechanisms widely used in 3-D models. More recently, Coates et al. (2015) compared MCM to simplified VOC mechanisms to look at ozone production for a selection of VOCs representative of urban air masses. Knote et al. (2015) conducted box model simulations using different chemical mechanisms and compared them to each other to understand mechanismspecific biases during a 3-D model intercomparison. Mazzuca et al. (2016) used an observation-constrained box model with a lumped carbon-bond mechanism to study photochemical oxidation and ozone production processes along a research aircraft campaign. Wolfe et al. (2016) presented a tool for 0-D atmospheric modeling that can use different chemical mechanisms and methods of photolysis frequency calculations.

This non-exhaustive list of recent studies using box models to study tropospheric chemistry shows the importance of and need for such tools. Box models are attractive due to their simplicity and low computational cost, but cannot provide a realistic representation of the entire atmosphere because they lack vertical and horizontal diffusion, boundary conditions, and numerous other processes that 3-D models take into account. In that regard, box model studies should not focus on replicating the most accurate predictions but rather aim at gaining significant fundamental and conceptual understanding of a given system of ordinary differential equations (ODEs), in the present case the one that governs tropospheric chemistry.

Another aspect of box models or reduced complexity models is the applicability to data assimilation research. Low dimension and/or box models are often used to design new data assimilation algorithms and to conceptually prove the advantage of a given method. In data assimilation theory, the use of the Lorenz model and other simple systems is common practice: Sandu et al. (2005) used a box model approach to design an adjoint-based sensitivity analysis for reactive gas-phase tropospheric chemistry. Ott et al. (2004) used a Lorenz-96 model to introduce a new formulation of the ensemble Kalman filter approach. Van Leeuwen (2010) also used the Lorenz model to investigate nonlinear advanced data assimilation techniques such as the particle filter.

Atmospheric composition data assimilation, and more generally inversion, is complex and computationally demanding. Reactive gas-phase photochemistry is highly nonlinear and has to deal with hundreds to thousands of variables. There is a need for a tool that allows for the exploration of suitable novel data assimilation approaches for atmospheric chemistry, assesses uncertainty and errors of a given chemical mechanism, and performs chemical sensitivity analysis from one parameter to another. All this should be possible with minimal computational expenses and coding skills required. In this paper, we present a new framework based on BOXMOX (BOX MOdel eXtension to KPP; Knote et al., 2015) called BEATBOX (Background Error Analysis
Testbed with Box Models) that is able to cycle data assimilation windows, calculate adjoint, ensemble, or even more advanced sensitivity analysis, and assess box model errors and uncertainties. In Sect. 2 we present in detail the structure of BEATBOX and its algorithms, as exemplified through case studies that we discuss in Sect. 3.

\section{Design of BEATBOX}

The Background Error Analysis Testbed with Box Models (BEATBOX) is a suite of tools that allows for a simple and fast investigation, comparison, and evaluation of any system of ordinary differential equations (ODEs) evolving over time. By "background error" we designate a general term for model error characterization. In this study, BEATBOX is used within the scope of atmospheric chemical mechanisms. Currently, the BEATBOX framework consists of a forecasting tool, the BOX MOdel eXtension (BOXMOX; Knote et al., 2015) to the Kinetic Pre-Processor (KPP; Sandu and Sander, 2006), presented in Sect. 2.1, and a data assimilation tool, which will be introduced in Sect. 2.2.

The BEATBOX structure is built upon observing system simulation experiments (OSSEs; Arnold and Dey, 1986). OSSEs are generally used in the field of numerical weather prediction (e.g., Kuo et al., 1998; Wang et al., 2008; Liu et al., 2009) and atmospheric composition and air quality predictions (e.g., Edwards et al., 2009; Claeyman et al., 2011; Barré et al., 2015, 2016). OSSEs allow for an assessment of the benefit of a potential new type of instrument for environmental predictions using a data assimilation system and are of crucial importance to define the requirements of a given instrument. Space agencies such as the National Aeronautics and Space Agency (NASA) and the European Space Agency (ESA) hence support OSSEs as tools to verify scientific readiness levels for proposed space missions. Also, the model and data assimilation requirements should be assessed to meet a required predictive capability. The current BEATBOX framework employs a box modeling approach to avoid the space dimension problem - the dimensionality of the problem is reduced to time and variables only. Hence the geometry, radiative specifications, and spatial resolution of a new instrument type are irrelevant. What can be easily explored within BEATBOX is the type of data assimilation method used (e.g., background error covariance calculations, localizations, inflation methods), the revisit time (or temporal sampling) of a measurement, the variable(s) observed, and straightforward comparisons between sets of ODEs (chemical schemes in our case).

A number of issues regarding the OSSE technique should be mentioned as well. Performing an OSSE could be costly in terms of setup, design, and computation. Numerical integration of the most state-of-the-art representation of the Earth system for sampling observations and benchmarking could be intensively costly and requires highly skilled staff and ex- 


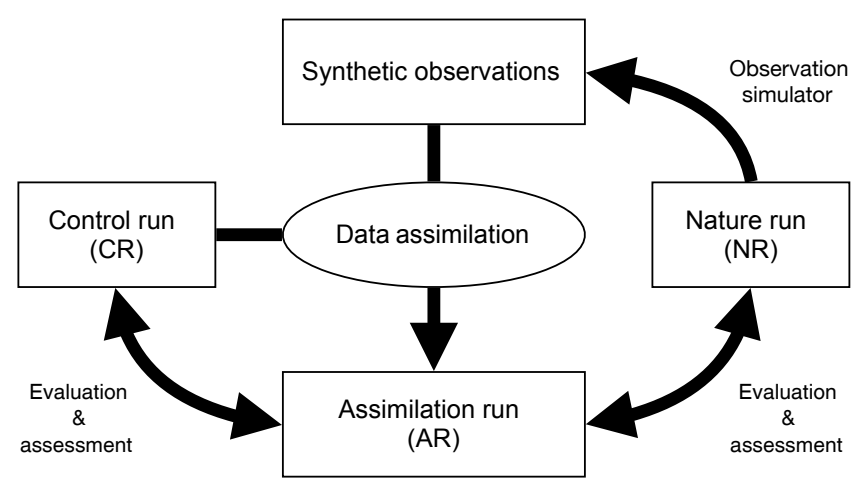

Figure 1. General flowchart of the BEATBOX system.

tensive collaboration between research entities. Approximations are often required to make experiments possible (e.g., the "identical twin" problem), necessitating careful diagnosis of the results that could limit scientific conclusions.

Ultimately an OSSE should be used to highlight model deficiencies and inaccuracies, and provide direct guidance for model improvement. In that context, BEATBOX could be considered as a derived OSSE framework focused on data assimilation techniques and model improvement rather than the benefit of new or future types of observations. Starting from a scientific question or hypothesis to be validated (or rejected) that fits the topics mentioned above, several components are required (Fig. 1):

- a nature run (NR) considered as the "true state" (the NR supposes to use the best model representation possible considering the state of the art; in this study, we used the Master Chemical Mechanism (MCMv3.3.1) as the NR);

- a control run (CR) as the prior estimated state of the atmosphere (compared to the NR, a simplified or degraded model should be used, for example a set of ODEs that can be implemented in large-scale 3-D models; in this study, we use MOZART-T1);

- an observation simulator that generates synthetic observation by sampling the NR (observation errors also need to be simulated);

- an assimilation run (AR) that is produced using the data assimilation tool merging the synthetic observation with the CR to produce the best estimate possible of the state; and

- a suite of diagnostic tools that use NR, CR, and AR designed to point out model and data assimilation technique limitations, ultimately providing a direct feedback for model improvement.

The BEATBOX framework has the capability to loop over several assimilation cycles, also called "cycling". Cycling with BEATBOX is schematically displayed in Fig. 2. Every cycle starts with the forecast step. By applying their respective model, the NR, the forecast $(F)$, and the $\mathrm{CR}$ are processed from cycle $t-1$ to cycle $t$. Then, $\mathrm{NR}_{t}$ is used to generate synthetic observations through the observation operator $H$ (see Sect. 2.2.2). These observations are assimilated into the forecast $F_{t}^{j}$ to produce the analysis $A_{t}^{j}$ with $j$ as the data assimilation method of choice using a gain $K^{j}$ (see Sects. 2.2.3-2.2.5). Then, $A_{t}^{j}$ will be used to generate new initial conditions $\mathrm{IC}_{t}^{j}$ to start a new forecast. $\mathrm{CR}_{t}$ serves as a reference to determine the performance of the assimilation method $j$ after $t$ forecast cycles. The forecast $F_{t}^{j}$ can be taken to quantify the skill of the assimilation method $j$ at the current cycle $t$. Afterwards, the cycle $t+1$ starts with its forecast step.

\subsection{Forecasting tool}

\subsubsection{The box model BOXMOX}

BOXMOX performs box model simulations with different chemical mechanisms using varying sets of input parameters. BOXMOX relies on KPP, a code generator that simplifies the numerical integration of systems of ODEs. The temporal evolution of concentrations of chemical compounds due to photochemistry is a prime example of such a system. KPP takes a predefined set of chemical equations (in our case a chemical mechanism) written in a symbolic, human-readable language and generates a computer code (FORTRAN, Matlab, C) containing a numerical solver to integrate the system over time. A number of integration methods are available (e.g., Rosenbrock or Runge-Kutta methods). In addition to predicting the evolution of concentrations over time, the resulting solver also delivers the Jacobian and Hessian matrices of the system. Adjoint models can be generated and tuned (Sandu et al., 2003) and the Jacobians of the adjoint of the model can be obtained (see Sect. 2.2.3). Building upon KPP, BOXMOX provides additional processes typically used in box model studies (emissions, photolysis, deposition, mixing) and allows for convenient data input. BOXMOX makes simulations of chamber experiments, Lagrangian-type air parcel studies, and a description of the chemistry in the atmospheric boundary layer feasible without effort. Input is done via simple text files: initial conditions, photolysis rates, temperature, boundary layer height, detrainment and entrainment, turbulent mixing, emission, and deposition are possible input parameters. BOXMOX is a stand-alone $\mathrm{C}$ and Fortran program running on Linux or Mac OS X. In this work we have extended BOXMOX with an interface written in the Python language (boxmox package) to interface more easily with BEATBOX.

BEATBOX uses BOXMOX to rapidly generate a large number of simulations with perturbed input parameters. The temperature, (time-varying) photolysis rates, and initial concentrations of each species included in the investigated chem- 


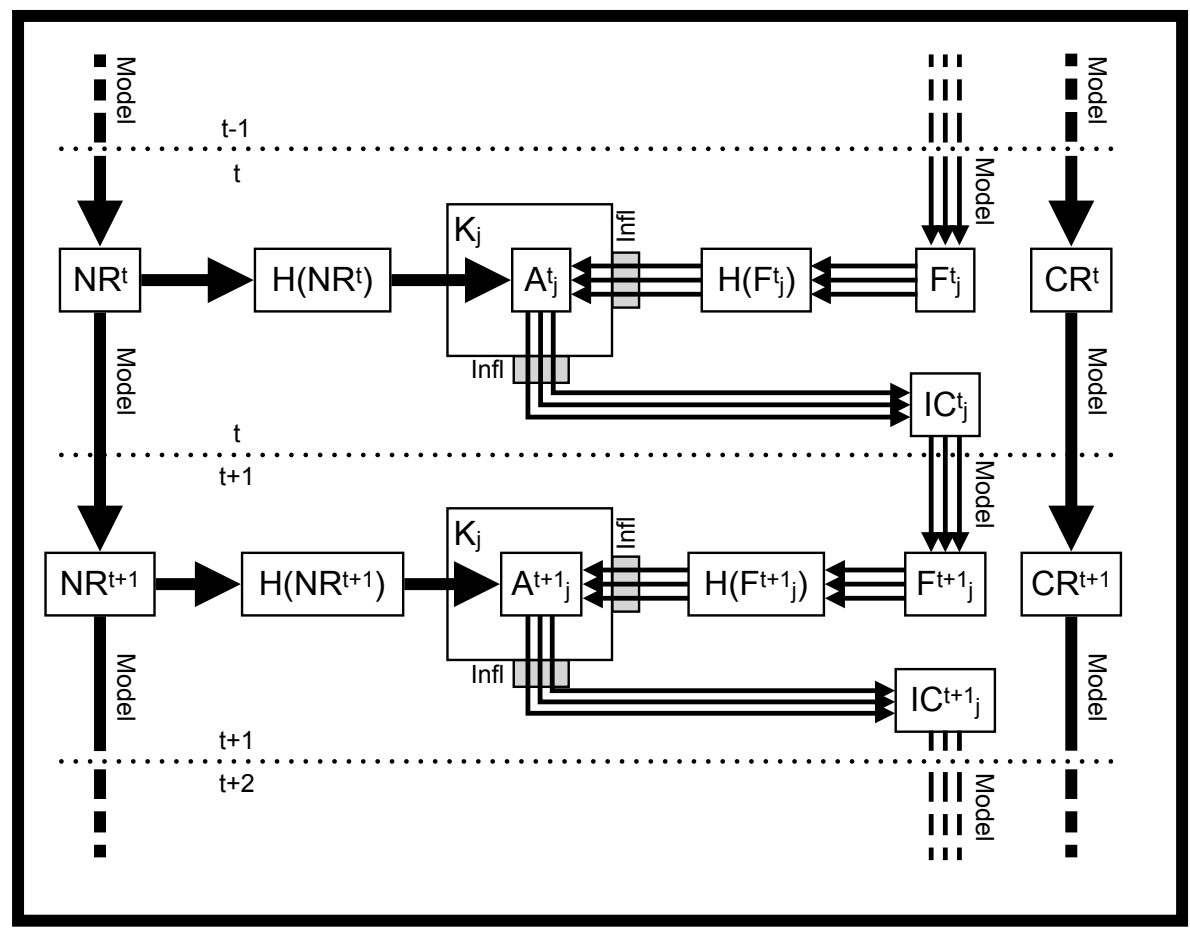

Figure 2. The cycling sequence with BEATBOX, with the assimilation window $t$, the assimilation method $j$, control run CR, nature run NR, observation operator $H$, gain $K$, analysis $A$, initial condition IC, and forecast $F$.

ical mechanism can be perturbed independently. Ensemble members can be generated by producing normal- or lognormal-distributed perturbation factors with the possibility to adjust the mean and the standard deviation for each perturbed variable. These perturbation factors are then multiplied by the relevant initial values to produce an ensemble of initial conditions.

In this work, we demonstrate the BEATBOX capabilities using MCMv3.3.1 as NR. Because of its near-explicit representation of atmospheric chemistry, MCMv3.3.1 is a good choice to be seen as the assumed "truth" within the context of an OSSE. The MOZART-T1 chemical mechanism is employed as the simplified and/or degraded model (CR, AR).

\subsubsection{Input data generation}

BOXMOX comes with a tool to generate input data from field campaign observations (the genbox Python package). Translation to mechanism-specific species naming and lumping is achieved using a translation tool (the chemspectranslator package) originally based on the emission database created by Bill Carter (UC Riverside; http: $/ /$ www.cert.ucr.edu/ carter/emitdb). The current system uses data collected in the FRAPPE (Front Range Air Pollution and Photochemistry Experiment) field campaign (frappedata package).

During FRAPPE, a number of flight measurements with remote sensing and in situ devices of numerous quanti- ties were performed, including concentrations of chemical species, photolysis rates, and temperature. In the examples shown we use measurements taken during FRAPPE with the NCAR C130 research aircraft to initialize the box model. Photolysis rates measured during FRAPPE are used in the examples shown here. For other cases in which photolysis rates are missing, we provide the ability to use photolysis rates calculated by the Tropospheric Ultraviolet Visible (TUV) radiation model version 5.1 (Madronich and Flocke, 1997) in BOXMOX using the tuv Python package.

In this current version of the code only measurements taken during the FRAPPE campaign have been used, but data from other field campaigns can be easily adapted as well with minimal code development.

\subsection{Data assimilation tool}

The beatboxtestbed Python package provides a data assimilation tool that samples observations from the NR, with the possibility of tuning the observation error parameters. By assimilating observations, different sensitivity analyses can be used, such as adjoint, ensemble, or combined (in this paper called hybrid), and are included in this version of BEATBOX (see Sects. 2.2.3-2.2.5). For notation purposes, we represent $x$ and $y$ as variables in model or state space and observation space, respectively. 


\subsubsection{The data assimilation problem}

Data assimilation combines observations and model information (also called forecast or background) to derive an optimal state (analysis) with a reduced error to provide the best initial condition for a subsequent forecast (see, e.g., Lahoz et al., 2010). Consider $n \in \mathbb{N}$ and $p \in \mathbb{N}$ the dimensions of the state (or model) space and the observation space, respectively. Following Nichols (2010) the solution to the data assimilation problem is commonly expressed as follows:

$\boldsymbol{x}_{\mathrm{a}}=\boldsymbol{x}_{\mathrm{b}}+\mathbf{K}\left(y_{\mathrm{o}}-H\left(x_{\mathrm{b}}\right)\right)$,

where $\boldsymbol{x}_{\mathrm{a}}$ is the analysis state, $\boldsymbol{x}_{\mathrm{b}}$ the background state, $y_{\mathrm{o}}$ the observation, $H$ the observation operator (also known as forward operator in the variational formalism), and $\mathbf{K}$ the gain matrix. The gain matrix $\mathbf{K}$ handles the transformation from the observation space to the state (or model) space. Conversely $H$ handles the transformation from model space to observation space. The above equation can also be expressed in the incremental form, such as

$\Delta \boldsymbol{x}=\mathbf{K} \Delta y$,

with $\Delta \boldsymbol{x}$ called the increment and $\Delta y$ called the innovation (or departure). The innovation in the observation space is then translated to the model space by applying the gain matrix K. Different methods exist to estimate the $\mathbf{K}$ gain; see Sects. 2.2.3-2.2.5. Commonly the gain matrix is given by

$\mathbf{K}=\mathbf{B} H^{\mathrm{T}}\left(H \mathbf{B} H^{\mathrm{T}}+\mathbf{R}\right)^{-1}$,

where $\mathbf{B}$ and $\mathbf{R}$ are the error covariance matrices of the background (or model) and observation, respectively. In the BEATBOX framework a single observation in a given assimilation window $(p=1)$ and only the dimension along the chemical variables is considered. Hence, $\mathbf{R}$ simplifies to a $1 \times 1$ matrix, a scalar $\sigma_{\mathrm{o}}^{2}$ the observation error variance, and $H \mathbf{B} H^{\mathrm{T}}$ also simplifies to a scalar $\sigma_{\mathrm{b}}^{2}$ (see Sect. 2.2.2 below) the background error variance in the observation space. Then $\mathbf{B} H^{\mathrm{T}}$ can be seen as $\sigma_{\mathrm{b}}^{2} \boldsymbol{s}$, where $\boldsymbol{s}$ can be called the sensitivity vector. The gain matrix in Eq. (3) then becomes a vector $\boldsymbol{\kappa}$ and can be reformulated as follows:

$\boldsymbol{\kappa}=\sigma_{\mathrm{b}}^{2}\left(\sigma_{\mathrm{b}}^{2}+\sigma_{\mathrm{o}}^{2}\right)^{-1} \boldsymbol{s}$

\subsubsection{Observation generator - synthetic observations}

Generating observations consists of the following steps:

- sampling values from the nature run,

- perturbing those values to simulate an observation inaccuracy, and

- specifying an observation error value.
BEATBOX forecasts have no dimensions in the 3-D atmospheric space (latitude, longitude, altitude). Sampling the NR to simulate observations is straightforward. Conventionally, $H$ is defined as the observation operator and handles the transformation of information defined in the model space to the observation space to compare model and observation quantities. Then, the $H$ operator can be expressed as $H^{\mathrm{T}}=[0,0, \ldots, 1, \ldots, 0]$. If no observation error is simulated the observation is defined as a perfect observation and the observation value is $y_{\mathrm{o}}=H\left(x_{\mathrm{NR}}\right)$.

If the observation error needs to be simulated, then $y_{0}$ is generated by adding a perturbation. In that version of BEATBOX the perturbation is assumed to be a normal distribution. But other probability density functions can be implemented easily (a lognormal perturbation is also implemented in this version). Then,

$y_{\mathrm{o}}=H\left(x_{\mathrm{NR}}\right)+N(M, \Sigma)$,

with $N(M, \Sigma)$ as a normal distribution of mean $M$ and standard deviation $\Sigma$. The latter two quantities can be viewed as the observation bias or accuracy $(M)$ and precision $(\Sigma)$. Finally, an associated observation error $\sigma_{\mathrm{o}}$ is associated with $y_{\mathrm{o}}$ for data assimilation; $\sigma_{\mathrm{o}}$ can account for bias and/or precision, overestimating, or underestimating those parameters depending on whether the effect of observation error needs to be tested in BEATBOX. In the following case study (see Sect. 3) we assume non-biased observation and non-biased observation error with a Gaussian distribution, leading to $\sigma_{\mathrm{o}}=\Sigma$.

\subsubsection{Adjoint sensitivity}

Adjoint sensitivities can be calculated using the KPP Jacobian matrix of output from the adjoint model at a given time step. In our case, we make the approximation that the change of the state $\boldsymbol{x}$ (that includes the observed variable $y$ ) at the time step $t$ relative to the change of the observed variable $y$ at a previous time step $t-1$ (i.e., $\mathrm{d} \boldsymbol{x}_{t} / \mathrm{d} y_{t-1}$ or $\mathrm{d} y_{t} / \mathrm{d} y_{t-1}$ ) is linear. In the current study, we assume $t-1$ and $t$ at the beginning and the end of the assimilation window. The adjoint sensitivity vector of the state $\boldsymbol{x}$ to a given observed variable $y$ at time $t$ can be computed using the Jacobian vectors as follows:

$\boldsymbol{s}=\frac{\mathrm{d} \boldsymbol{x}_{t}}{\mathrm{~d} y_{t}}=\frac{\mathrm{d} \boldsymbol{x}_{t}}{\mathrm{~d} y_{t-1}} \cdot\left(\frac{\mathrm{d} y_{t}}{\mathrm{~d} y_{t-1}}\right)^{-1}$.

The adjoint assimilation method runs a single forecast with the forward model and also runs the adjoint model and computes the analysis by combining Eqs. (1), (4), and (6). The innovation in observation space $\Delta y$ is calculated and the state $\boldsymbol{x}$ is inferred using the $\boldsymbol{\kappa}$ gain that includes the adjoint sensitivity $\boldsymbol{s}$. In this method, $\sigma_{\mathrm{b}}$ should be determined with ad hoc information and will not change during the cycling process. However, different methods exist to scale $\sigma_{\mathrm{b}}$ appropriately 
between each cycle. In the BEATBOX context this can be further explored using the provided OSSE framework.

\subsubsection{Ensemble sensitivity}

Ensemble methods run a perturbed set (ensemble) of model realizations in parallel and derive model error and sensitivity using the created ensemble. This gives multiple realizations $i$ of the model in the observation space $y_{i}$ and model space $\boldsymbol{x}_{i}$. The standard deviation of the ensemble (or the ensemble spread) is used to estimate $\sigma_{\mathrm{b}}$ in the observation space, such as $\sigma_{y}=\sqrt{E\left[\left(y_{i}-E\left[y_{i}\right]\right)^{2}\right]}=\sigma_{\mathrm{b}}$ with $E$ as the averaging operator. Similarly, the ensemble spread in model space can be estimated as $\sigma_{x}=\sqrt{E\left[\left(\boldsymbol{x}_{i}-E\left[\boldsymbol{x}_{i}\right]\right)^{2}\right]}$. Then statistical assumptions are made to derive the sensitivity between $y$ and $\boldsymbol{x}$. One of the commonly used methods as described by Anderson (2003) is to draw a linear fit in the least-squares sense between $y$ and $\boldsymbol{x}$ using the ensemble members, such as

$\boldsymbol{s}=\left(\sum \boldsymbol{x}_{i} y_{i}\right)\left(\sum y_{i} y_{i}\right)^{-1}=\frac{\boldsymbol{\sigma}_{x y}}{\sigma_{y}^{2}}=\boldsymbol{r}_{\boldsymbol{x} y} \circ \frac{\boldsymbol{\sigma}_{x}}{\sigma_{y}}$,

where $\boldsymbol{r}_{x y}$ and $\boldsymbol{\sigma}_{x y}$ are respectively the correlation coefficient and covariance vectors between $y$ and each chemical variable of $\boldsymbol{x}$ and $\circ$ is the Schur product operator. Then, the innovation in the observation space $\Delta y_{i}$ is calculated for each ensemble member. The ensemble state vector $\boldsymbol{x}_{i}$ is inferred using the same $\boldsymbol{k}$ gain that includes the same ensemble sensitivity $\boldsymbol{s}$ for each ensemble member.

\subsubsection{Hybrid sensitivity and further approaches}

In the current version of BEATBOX the hybrid sensitivity is defined as a combination between the two approaches mentioned above: it uses an adjoint sensitivity for each ensemble member, such as

$\boldsymbol{s}_{i}=\frac{\mathrm{d} \boldsymbol{x}_{t, i}}{\mathrm{~d} y_{t, i}}=\frac{\mathrm{d} \boldsymbol{x}_{t, i}}{\mathrm{~d} y_{t-1, i}} \cdot\left(\frac{\mathrm{d} y_{t, i}}{\mathrm{~d} y_{t-1, i}}\right)^{-1}$.

In that sense, each ensemble member has its own adjoint sensitivity calculation $\boldsymbol{s}_{i}$ and its own innovation in observation space $\Delta y_{i}$. This results in an independent or different inference on the state vector for each ensemble member $\boldsymbol{x}_{i}$. This method is just an example of what can be explored with the BEATBOX system. Because of the simplicity of the code and the low dimensionality of the problem, advanced techniques can be easily implemented and analyzed. Other hybrid methods and filters, such as a polynomial filter or particle filters and their benefits for highly nonlinear systems, can be explored with ease with the proposed framework as well.

\subsubsection{Inflation}

For ensemble methods, to avoid the filter divergence problem (Fitzgerald, 1971), inflation algorithms are needed. In the BEATBOX framework we included the inflation method as proposed by Anderson (2007), such as

$\boldsymbol{x}_{i}^{\mathrm{infl}}=\sqrt{\lambda}\left(\boldsymbol{x}_{i}-E\left[\boldsymbol{x}_{i}\right]\right)+E\left[\boldsymbol{x}_{i}\right]$,

with $\lambda$ called the covariance inflation factor that determines how much the ensemble members $\boldsymbol{x}_{i}$ are spread out from the mean $E[x]$. Many different methods exist to estimate $\lambda$; it can be chosen as constant over time or adaptive given the ensemble spread in observation space $\sigma_{\mathrm{b}}$, observation error $\sigma_{\mathrm{o}}$, and the innovation norm from the ensemble mean $\theta=$ $\left|E\left[y_{i}\right]-y_{\mathrm{o}}\right|$. In the version of BEATBOX presented here we calculate $\lambda$ as

$\lambda=\frac{\theta^{2}-\sigma_{\mathrm{o}}^{2}}{\sigma_{\mathrm{b}}^{2}}$.

If $\lambda$ is found to be smaller than 1 , the ensemble spread is assumed to be large enough and no inflation is calculated. Straightforward computation of $\lambda$ as above assumes linearity between observation space and model space, which is true in the current BEATBOX framework. More advanced ways to compute $\lambda$ as described in Anderson (2007, Appendix A) have been tested and implemented in BEATBOX and can be used as well. Finally, to conserve the positive definite nature of the ensembles and also prevent forcing ensemble members to zero that would otherwise be inflated to negative values, we reduce the inflation factor iteratively on every value of the state:

$\lambda \leq\left(\frac{E\left[x_{i}\right]}{x_{i}^{\text {infl }}-E\left[x_{i}\right]}\right)^{2}$.

This is one of several methods to conserve the physical aspects of the ensemble: it might under-disperse the ensemble in some cases of low concentrations but ensures that the inflation is kept to physical values. In the BEATBOX framework, users can easily implement and explore new inflation methods for nonlinear, definite positive, and perturbation of highly sensitive systems, as in the case of reactive gas-phase chemistry.

\subsubsection{Localization}

One should consider to what extent the sensitivities should be relied on. Localization algorithms try to limit the impact on the analysis of errors in the sample covariance between observations and model state variables (Mitchell and Houtekamer, 2000). Depending on the ensemble size or the mathematical assumptions to compute a sensitivity, a localization function $\mathbf{C}$ should be used to define where to apply the sensitivity $\boldsymbol{s}$, such as

$\boldsymbol{s}_{\mathrm{loc}}=\boldsymbol{s} \circ \mathbf{C}$.

In the current BEATBOX framework, it is possible to specify which species should be inferred, e.g., $\mathbf{C}^{\mathrm{T}}=$ $[0,1, \ldots, 1, \ldots, 0]$. 


\subsection{Flux method for model analysis}

A flux tool has been included in boxmox that calculates the production and loss fluxes for a given chemical component. Consider the generalized chemical reactions, such as

$\sum_{i}[R]_{i} \stackrel{k}{\longrightarrow} \sum_{j}[P]_{j}$

with $k$ called the rate constant. The chemical flux can be expressed as

$$
\frac{d[\mathbf{R}]}{d t}=-\frac{d[\mathbf{P}]}{d t}=-k \prod_{i}[R]_{i}=k \prod_{j}[P]_{j} .
$$

For a given chemical component, a detailed budget of chemical production and loss can be made by identifying different chemical reactions. An example of the application of the flux tool is shown in Sect. 3.4.

\section{Application to an urban pollution case study}

In this section, we provide an example case study to showcase the capabilities of the BEATBOX framework.

\subsection{Control run and nature run}

Temperature, concentrations, and photolysis rates from the FRAPPE data (see Sect. 2.1.2) are used for initial conditions. In the present simulations, all environmental parameters such as temperature and photolysis rates are kept constant. Interactions between the simulated box and the surrounding are neglected, and the box model simulation can be seen as "chemistry in a jar" similar to a chamber experiment without consideration of wall losses, in which only the temporal evolutions due to chemical reactions are allowed. Initial conditions for primary VOCs and inorganic compounds are provided using the FRAPPE observations.

The present case study focuses on an air mass originating from the industrial area of Commerce City near Denver, Colorado. Figure 3 shows the estimated temporal evolution over the first $60 \mathrm{~h}$ of simulation for the $\mathrm{VOC} / \mathrm{NO}_{x}$ $\left(\mathrm{NO}_{x}=\mathrm{NO}+\mathrm{NO}_{2}\right)$ and toluene / benzene ratio using the MOZART-T1 scheme. The vertical lines suggest the VOClimited ( $\mathrm{VOC} / \mathrm{NO}_{x}$ ratio $\left.<=4\right), \mathrm{NO}_{x}$-limited $\left(\mathrm{VOC} / \mathrm{NO}_{x}\right.$ ratio $>15)$, and transition region $\left(4<\mathrm{VOC} / \mathrm{NO}_{x}\right.$ ratio $<=15$ ) to show that the simulation transitions through different $\mathrm{O}_{3}$ production regimes with possibly very different relevant chemical pathways. The measurements show an initially strongly VOC-limited air indicative of an urban air mass. The $\mathrm{VOC} / \mathrm{NO}_{x}$ ratio of the aging air increases in time. During the first $15 \mathrm{~h}$ the simulation shows a strong VOC-limited regime. A transition regime spans from $15 \mathrm{~h}$ to approximately $30 \mathrm{~h}$. After the transition period the chemical regime becomes $\mathrm{NO}_{x}$ limited, which is representative of

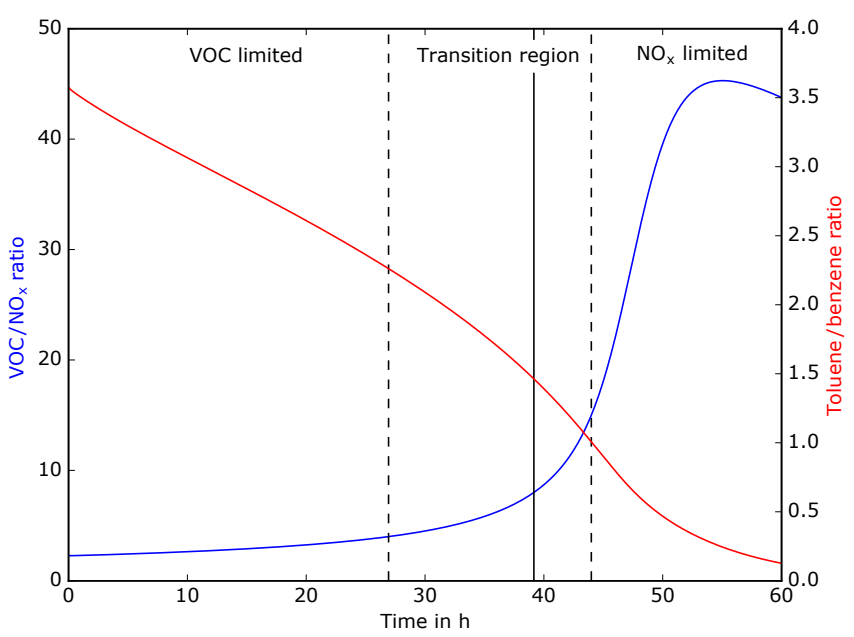

Figure 3. Temporal evolution of the $\mathrm{VOC} / \mathrm{NO}_{x}$ (blue line) and toluene / benzene (red line) ratios over $60 \mathrm{~h}$ derived from MOZART-T1 (CR).

more rural or background conditions. The toluene / benzene ratio is used as a qualitative measure of photochemical age. Toluene and benzene are considered to have the same sources but toluene is more quickly oxidized than benzene, which leads to a decline in the ratio over time.

The temporal evolutions of some key species in the NR and the CR are displayed in Fig. 4. Nitrogen dioxide $\left(\mathrm{NO}_{2}\right)$ shows a decrease over time, with a stronger decay in the NR than in the $\mathrm{CR}$. Ozone $\left(\mathrm{O}_{3}\right)$ production is observed over time with a therefore stronger production in the NR than in the $\mathrm{CR}$. The hydroxyl-radical $(\mathrm{OH})$ availability increases over time, especially between 20 and $35 \mathrm{~h}$, which can be identified as the end of the transition from a VOC-limited to a $\mathrm{NO}_{x}$-limited regime. In the VOC-limited and $\mathrm{NO}_{x}$-limited regimes, the $\mathrm{OH}$ increase is smaller. Formaldehyde $\left(\mathrm{CH}_{2} \mathrm{O}\right)$ shows in both NR and CR a decrease followed by an increase and finally a decrease. Those variations illustrate the change of chemical regimes from VOC limited to transition to $\mathrm{NO}_{x}$ limited. If we compare the NR to the CR the change of chemical regimes is lagged. The change of regimes seems to occur faster in the NR than in the $\mathrm{CR}$, suggesting different reactivity and pathways of oxidation in the NR than in the CR.

\subsection{Assimilation runs}

We focus on assimilating $\mathrm{NO}_{2}$ and $\mathrm{CH}_{2} \mathrm{O}$ concentrations in two separate experiments to show the capabilities of the BEATBOX framework. This is motivated by the fact that $\mathrm{NO}_{2}$ and $\mathrm{CH}_{2} \mathrm{O}$ play a key role in atmospheric chemistry, especially for short-lived chemical compounds. Also, $\mathrm{NO}_{2}$ and $\mathrm{CH}_{2} \mathrm{O}$ are, and will be, observed from space from both low-Earth and geostationary orbiters and from in situ observations. We define a reduced state vector for simplicity of the demonstration with the species described above: $\mathrm{NO}_{2}, \mathrm{O}_{3}$, $\mathrm{CH}_{2} \mathrm{O}$, and $\mathrm{OH}$. 

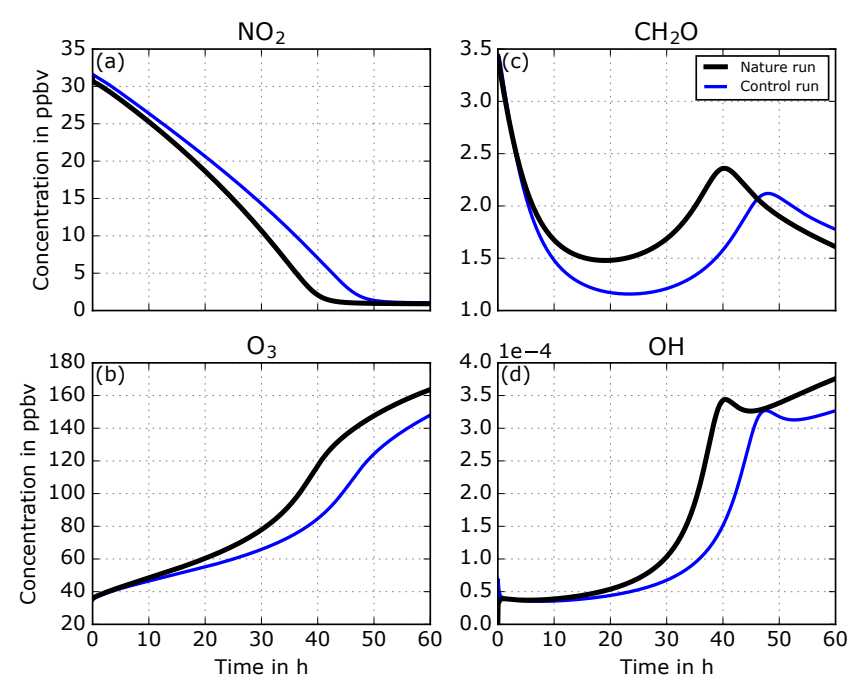

Figure 4. Time series of concentrations of $\mathrm{NO}_{2}, \mathrm{O}_{3}, \mathrm{CH}_{2} \mathrm{O}$, and $\mathrm{OH}$ over $60 \mathrm{~h}$ from the control run (MOZART-T1, blue lines) and from the nature run (MCMv3.3.1, black lines).

We use an assimilation window of $3 \mathrm{~h}$. Unbiased observations have been defined and assumed to be at the end of the assimilation window with an observation error of $0.75 \mathrm{ppbv}$ for $\mathrm{NO}_{2}$ and $0.07 \mathrm{ppbv}$ for $\mathrm{CH}_{2} \mathrm{O}$ (corresponding to approximately 2.5 and $5 \%$ of the concentrations at initial time). All the generated observations have well-estimated observation error, such as $\sigma_{\mathrm{o}}=\Sigma$ (see Sect. 2.2.2). The model error in the observation space $\sigma_{\mathrm{b}}$ has to be specified for the adjoint technique and is set to $2.5 \%$ for $\mathrm{NO}_{2}$ and $5 \%$ for $\mathrm{CH}_{2} \mathrm{O}$ of the current forecast concentration (see Sect. 2.2.3). For the ensemble method, the ensemble spread implicitly defines $\sigma_{\mathrm{b}}$ using 50 ensemble members that are generated by perturbing the initial $\mathrm{NO}_{2}$ concentration by $2.5 \%$ for $\mathrm{NO}_{2}$ assimilation and by perturbing the initial $\mathrm{CH}_{2} \mathrm{O}$ concentration by $5 \%$ for $\mathrm{CH}_{2} \mathrm{O}$ assimilation (see Sect. 2.2.4). Adaptive inflation is applied on the ensembles at every assimilation window to maintain a realistic ensemble spread to weight the observation and model appropriately (see Sect. 2.2.6).

\subsection{1 $\mathrm{NO}_{2}$ assimilation}

We assimilate $\mathrm{NO}_{2}$ observations using two different localizations. The first experiment infers only $\mathrm{NO}_{2}$ concentration and the second infers the entire state vector (i.e., $\mathrm{NO}_{2}, \mathrm{O}_{3}$, $\mathrm{CH}_{2} \mathrm{O}$, and $\mathrm{OH}$ concentrations). After looking at the evolution of $\mathrm{NO}_{2}$ concentrations (see Fig. 5), all three assimilation methods in both experiments (adjoint, ensemble, and hybrid) tend to move the analysis closer toward the observations and hence the NR. Some differences among the three ARs can be found. Recalling Sects. 2.2.1 and 2.2.2, because of the dimensionality of the problem the sensitivity from observation space $\mathrm{NO}_{2}$ to the model variable $\mathrm{NO}_{2}$ is equal to identity, $s=1$, for every assimilation method. Hence, the difference among assimilation runs will only result from differences in $\boldsymbol{x}_{\mathrm{b}}$ and $\sigma_{\mathrm{b}}$ after a subsequent forecast. The ensemble and hybrid methods show a quicker improvement than the single member adjoint method due to the adaptive nature of $\sigma_{\mathrm{b}}$ with the inflation (see Sect. 2.2.6). The single adjoint method keeps $\sigma_{\mathrm{b}}=2.5 \%$, while the ensemble and hybrid methods can tune this value with the inflation.

When only $\mathrm{NO}_{2}$ is inferred the other species are modified and this could be called the model response to assimilated changes: $\mathrm{NO}_{2}$ is decreased, which increases the $\mathrm{OH}$ availability for other oxidation pathways. A slight increase in $\mathrm{O}_{3}$ is noted and more significantly for $\mathrm{CH}_{2} \mathrm{O}$ during the transition region. The ensemble methods have a stronger effect due to the adaptive nature of $\sigma_{\mathrm{b}}$. The adjustment of $\mathrm{NO}_{2}$ concentration towards $\mathrm{NO}_{2}$ observation can be more effective and this can have a stronger effect on the model response.

When the entire state vector is inferred, i.e., no localization, slight additional increases occur in $\mathrm{O}_{3}, \mathrm{CH}_{2} \mathrm{O}$, and $\mathrm{OH}$ in the transition region. In general, in the first 10 to $15 \mathrm{~h}$ when VOC-limited conditions dominate (see Fig. 3), the impact of $\mathrm{NO}_{2}$ assimilation is low. By definition, VOC-limited air is insensitive to changes in $\mathrm{NO}_{2}$, so if the model (CR) predicts VOC-limited conditions either adjoint sensitivities or ensemble sensitivity will remain small. After $15 \mathrm{~h}$ of simulation, the chemical regime transitions significantly to $\mathrm{NO}_{x}$ limited with higher sensitivity of the state to changes in $\mathrm{NO}_{2}$. After $40 \mathrm{~h}, \mathrm{NO}_{2}$ concentrations drop to very low values, $\mathrm{NO}_{2}$ assimilation increments are very small, and hence almost no inference on the other state variables is observed.

In the case study, inference from $\mathrm{NO}_{2}$ observation on the rest of vector is not the major reason for improvement. Model response from $\mathrm{NO}_{2}$ changes is mostly responsible for improving the state. $\mathrm{NO}_{x}$ concentrations drive the chemistry in the transition region and $\mathrm{NO}_{x}$-limited regimes. $\mathrm{NO}_{x}$ chemistry is well known and similarly represented between the NR and the CR. Hence the model response is likely to improve the state and not likely to produce additional errors.

\subsection{2 $\mathrm{CH}_{2} \mathrm{O}$ assimilation}

We repeat the experiments presented in Sect. 3.2.1, but assimilate $\mathrm{CH}_{2} \mathrm{O}$ observations instead of $\mathrm{NO}_{2}$. Figure $6 \mathrm{a}-\mathrm{d}$ show the concentration evolution when only $\mathrm{CH}_{2} \mathrm{O}$ is inferred. In the first $40 \mathrm{~h} \mathrm{CH}_{2} \mathrm{O}$ is underestimated by the $\mathrm{CR}$ mechanism. All three ARs show very similar results. During the analysis phases the $\mathrm{CH}_{2} \mathrm{O}$ concentrations are pushed up towards the NR. Those abrupt increases are systematically compensated for by the chemical mechanism nudging the system back into chemical equilibrium, i.e., towards the $\mathrm{CR}$ concentrations. This makes the $\mathrm{CH}_{2} \mathrm{O}$ concentration evolution a sawtooth shape. $\mathrm{CH}_{2} \mathrm{O}$ is a short-lived chemical compound (shorter than $\mathrm{NO}_{2}$ in this case study) and it is mainly driven by other chemical species concentrations. Ultimately $\mathrm{CH}_{2} \mathrm{O}$ concentrations in the ARs are slightly elevated after each $3 \mathrm{~h}$ forecast. These changes will very mildly affect the 
Only $\mathrm{NO}_{2}$ inferred
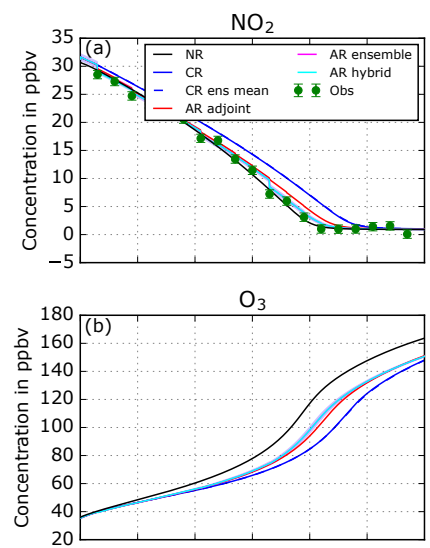

$\mathrm{CH}_{2} \mathrm{O}$
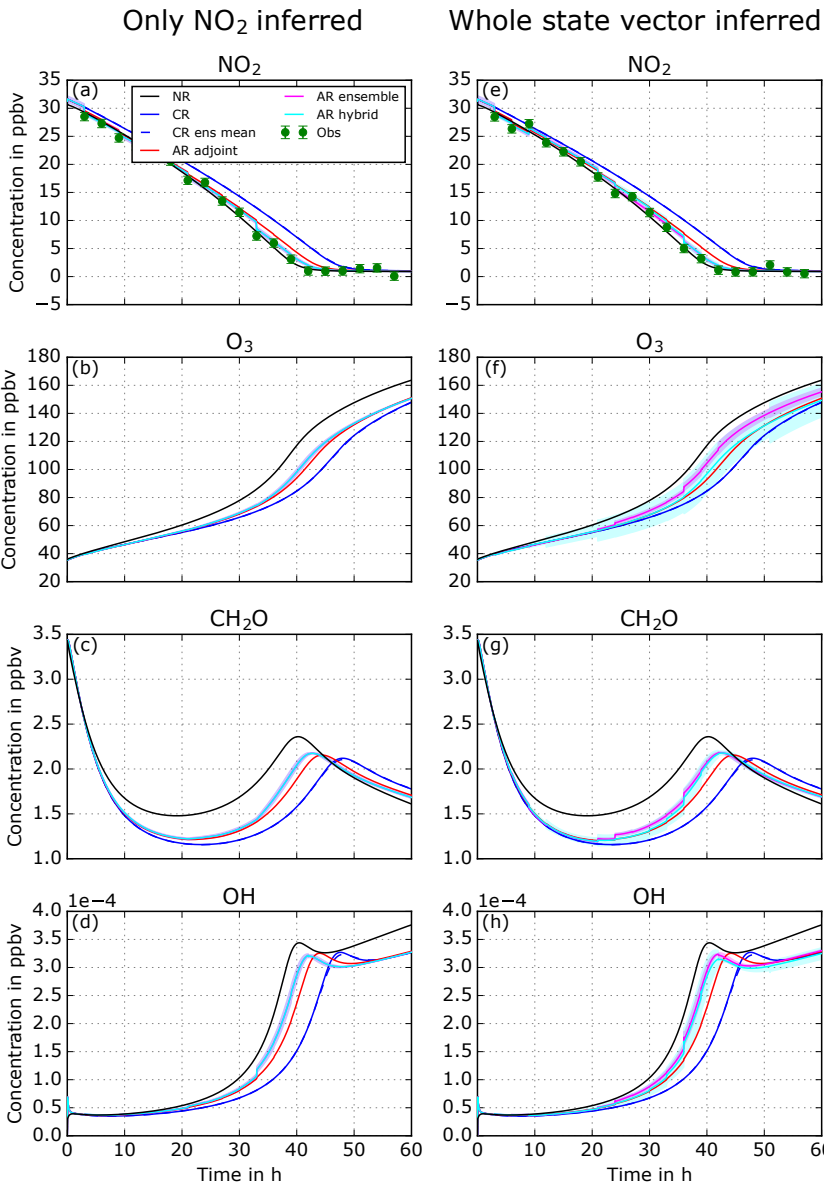

$\mathrm{CH}_{2} \mathrm{O}$
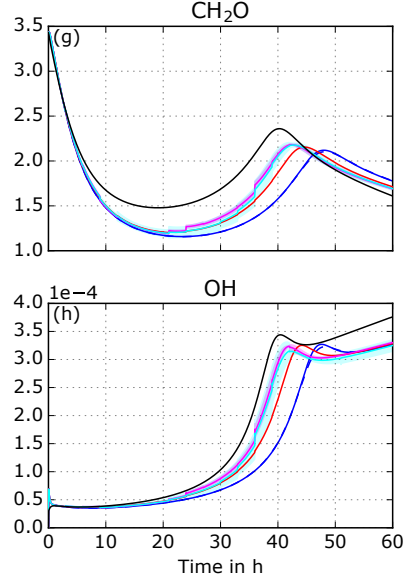

Figure 5. Evolution of the state vector over $60 \mathrm{~h}$, including the nature run (NR, black line), control run (CR, blue), and assimilation runs using adjoint (red), ensemble (pink), and hybrid (turquoise) methods. Two experiments with different localizations are displayed: only $\mathrm{NO}_{2}$ inferred (a-d) and whole state vector inferred (eh). Shaded areas show corresponding ensemble spread for ensemble and hybrid methods.

state vector concentrations. The model response to $\mathrm{CH}_{2} \mathrm{O}$ changes in $\mathrm{NO}_{2}, \mathrm{O}_{3}$, and $\mathrm{OH}$ is slight and definitely smaller than the model response to $\mathrm{NO}_{2}$ changes (see Sect. 3.2.1).

When the entire state vector is inferred, i.e., no localization (Fig. 6e-h), we observe very different results. The two ARs that are using adjoint sensitivities (hybrid and adjoint) show similar results as the previous experiment, and the sawtooth shape is still observed. The analysis shows strong increases in $\mathrm{CH}_{2} \mathrm{O}$ and the chemical mechanism tries to come back to the CR state. The inference on the other species of the state vector is noticeable, and $\mathrm{NO}_{2}, \mathrm{O}_{3}$, and $\mathrm{OH}$ concentrations are improved compared to the experiment when only $\mathrm{NO}_{2}$ is inferred.

In the ensemble method in which no localization is applied, the improvement in $\mathrm{CH}_{2} \mathrm{O}$ is of a different nature. The sawtooth behavior of the AR is not observed anymore and the chemical mechanism now seems to have changed
Only $\mathrm{CH}_{2} \mathrm{O}$ inferred

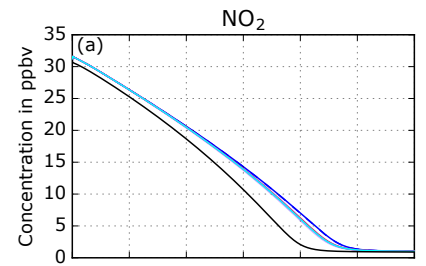

$\mathrm{O}_{3}$
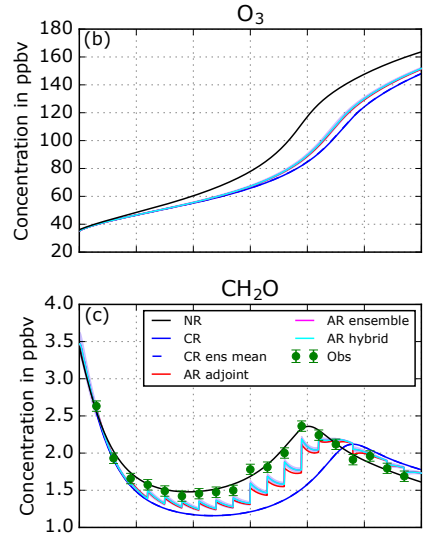

$\mathrm{OH}$

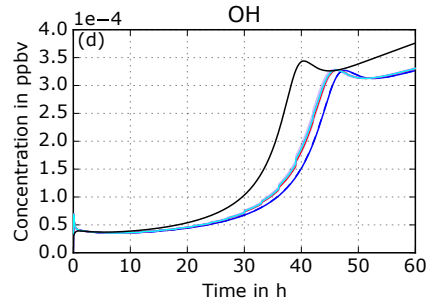

Figure 6. Same as Fig. 5 but with $\mathrm{CH}_{2} \mathrm{O}$ observations assimilated.

from systematic $\mathrm{CH}_{2} \mathrm{O}$ loss to $\mathrm{CH}_{2} \mathrm{O}$ production in the forecast. This will drive the AR to better fit the observations and hence the NR. The inference on other species shows significant changes that will in general change the sign of the error and sometimes increase it; $\mathrm{NO}_{2}$ becomes underestimated in the transition region, $\mathrm{O}_{3}$ is overestimated but then underestimated after $35 \mathrm{~h}$, and $\mathrm{OH}$ is overestimated in the transition region. In the ensemble method for the case study, the computed sensitivities seem significantly different from the adjoint sensitivities. This will allow us to change the chemical production and loss rate of $\mathrm{CH}_{2} \mathrm{O}$ to better adjust the $\mathrm{CH}_{2} \mathrm{O}$ concentration, but at the risk of disturbing the rest of the state vector and increasing errors that might lead to unphysical results. We diagnose the difference among sensitivities from the two experiments presented above in Sect. 3.3. We also diagnose the chemical behavior change from $\mathrm{CH}_{2} \mathrm{O}$ assimilation using fluxes in Sect. 3.4.

\subsection{Sensitivities of the off-diagonal elements of the background error covariance matrix}

Figure 7 shows the sensitivities of the unobserved species to a change in $\mathrm{NO}_{2}$ and $\mathrm{CH}_{2} \mathrm{O}$, respectively, at the end of each $3 \mathrm{~h}$ 
assimilation window with the single member adjoint and the ensemble method (see Sects. 2.2.3 and 2.2.4). To make sensitivities comparable we have normalized (divided) them by the state concentrations. For example, $\boldsymbol{s}\left(\mathrm{NO}_{2}, \mathrm{O}_{3}\right)$, the sensitivity of $\mathrm{NO}_{2}$ changes over $\mathrm{O}_{3}$, will most likely be orders of magnitude larger than $\boldsymbol{s}\left(\mathrm{NO}_{2}, \mathrm{OH}\right)$ since $\mathrm{O}_{3}$ concentrations are orders of magnitude larger than $\mathrm{OH}$ concentrations.

For $\mathrm{NO}_{2}$ assimilation, both methods deliver similar results. The sensitivities are in general small, not above $60 \%$. Sensitivities are small or negligible in the VOC-limited regime but become more significant during the transition and the $\mathrm{NO}_{x}$-limited regimes, mostly after $30 \mathrm{~h}$. The changes in $\mathrm{NO}_{2}$ from assimilation after $30 \mathrm{~h}$ are rather small and hence the inference on other species will be small. This then confirms that the most important part of the changes from $\mathrm{NO}_{2}$ assimilation is due to the model response from $\mathrm{NO}_{2}$ changes and secondly due to the data assimilation inference on the other species of the state.

For $\mathrm{CH}_{2} \mathrm{O}$ assimilation we saw significant differences in behavior between adjoint and ensemble methods to compute the sensitivities. The adjoint displays rather small sensitivities, peaking at $20 \%$ but mostly below $10 \%$. Those sensitives are observed during the VOC-limited regime when the chemistry is sensitive to changes from VOC concentrations and disappear during the transition region and become insignificant in the $\mathrm{NO}_{x}$-limited regime. This explains the small but reasonable impacts from $\mathrm{CH}_{2} \mathrm{O}$ changes on the rest of the state. Concerning the ensemble method, the computed sensitivities are much larger and do not decrease after the transition regime. Values switch abruptly from negative to positive and are sometimes above $200 \%$. To understand this unphysical behavior, we display tracer-tracer relationships during the assimilation phase of the ninth cycle $(27 \mathrm{~h})$ in Fig. 8.

In Sect. 2.2.4, we defined the ensemble method sensitivity computation as a linear fit to the ensemble distribution between two species. Most state-of-the-art EnKF methods use this approximation. One can see the limitation to such a linear assumption after looking carefully at Fig. 8a and b. The prior and posterior states of the $\mathrm{NO}_{2}-\mathrm{O}_{3}$ distributions are displayed. The ensemble method represents the observation well; the ensemble distribution is at the $\mathrm{NO}_{2}$ level of the observation and the ensemble spread fits the observation error (green vertical bars). However, the relationship between $\mathrm{NO}_{2}$ and $\mathrm{O}_{3}$ that has formed during the ensemble AR after nine cycles is strongly curved and nonlinear, which is difficult to represent through the linear fit of the ensemble sensitivity. In this example, moving the ensemble members along the linear fit is moving the $\mathrm{O}_{3}$ distribution slightly away from the NR. At the same time the adjoint sensitivities are in comparison very weak (the slope is along the $y$ axis), slightly improving the state distributions but not very strongly. Finally, the hybrid distribution shows a larger spread and different distribution shape than the ensemble distribution. The adjoint inference does not strongly change the rest of the state, which maintains the chemical production and loss rates of

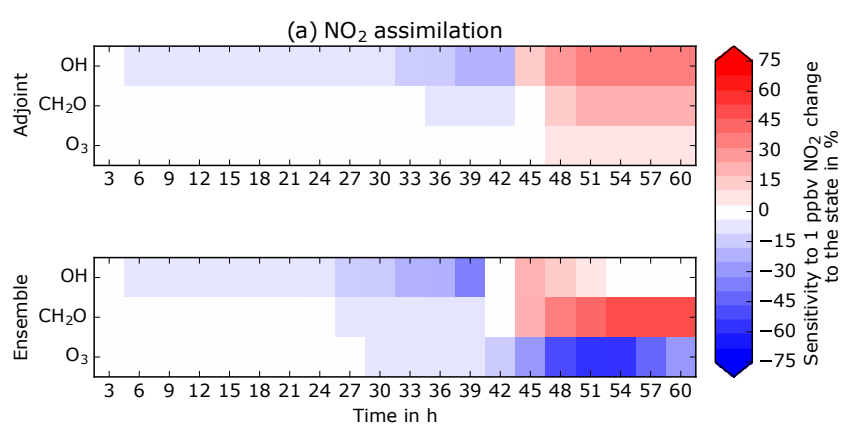

(b) $\mathrm{CH}_{2} \mathrm{O}$ assimilation

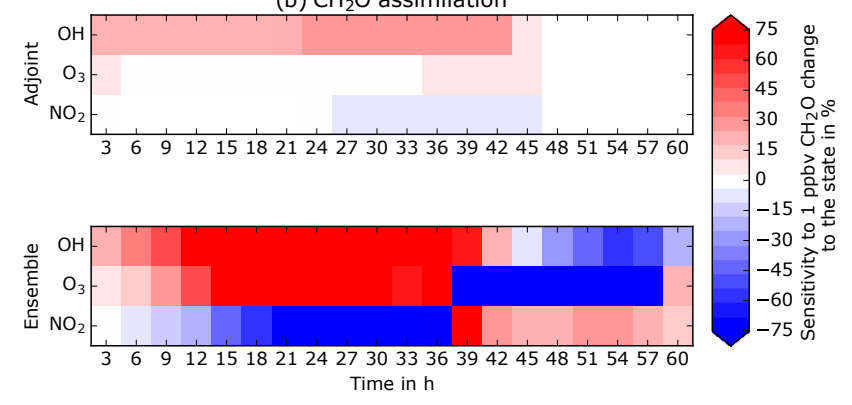

Figure 7. Temporal evolution of the sensitivities of the unobserved state species to changes from the observed one at the end of each $3 \mathrm{~h}$ assimilation for $\mathrm{NO}_{2}$ assimilation (a) and $\mathrm{CH}_{2} \mathrm{O}$ assimilation (b).

$\mathrm{CH}_{2} \mathrm{O}$ that fall into a temporary attractor; the model wants to go back to the CR concentrations and creates a sawtoothshaped concentration evolution over time (see Sect. 3.2.2). After $3 \mathrm{~h}$, the forecast will be significantly far from the observation and the distribution will be inflated. The ensemble inference strongly changes the rest of the state, which will change the chemical production and loss rates and drive the system out of the attractor. To understand this more clearly, the flux tool is presented in the following section.

\subsection{Model diagnostics using fluxes}

In this last section, we will focus on the $\mathrm{CH}_{2} \mathrm{O}$ fluxes (production and loss) over the ninth cycle forecast ( 25 to $27 \mathrm{~h}$ ) of the $\mathrm{CH}_{2} \mathrm{O}$ assimilation without localization. In Fig. 9 are the individual contributions of production and loss of $\mathrm{CH}_{2} \mathrm{O}$ for the NR and the same member of the $\mathrm{CR}$, AR ensemble, and AR hybrid. The flux tool isolates a few different reactions with NR because the chemical scheme is different and more detailed than the CR. In this case study, $\mathrm{CH}_{3} \mathrm{O} \rightarrow \mathrm{CH}_{2} \mathrm{O}+\mathrm{HO}_{2}$ in the NR is simply an intermediate reaction of $\mathrm{NO}+\mathrm{CH}_{3} \mathrm{O}_{2} \rightarrow \mathrm{CH}_{2} \mathrm{O}+\mathrm{NO}_{2}+\mathrm{HO}_{2}$. Other than this reaction, the isolated reactions are similar.

The NR fluxes to the CR fluxes show the same order of production and loss importance; however, the NR fluxes are stronger. Overall the loss terms in the NR are stronger than the $\mathrm{CR}$, leading to a net chemical flux that is negative. The CR rates are significantly faster than the AR ensemble rates, and the slopes of the rates (i.e., the second derivative of the 

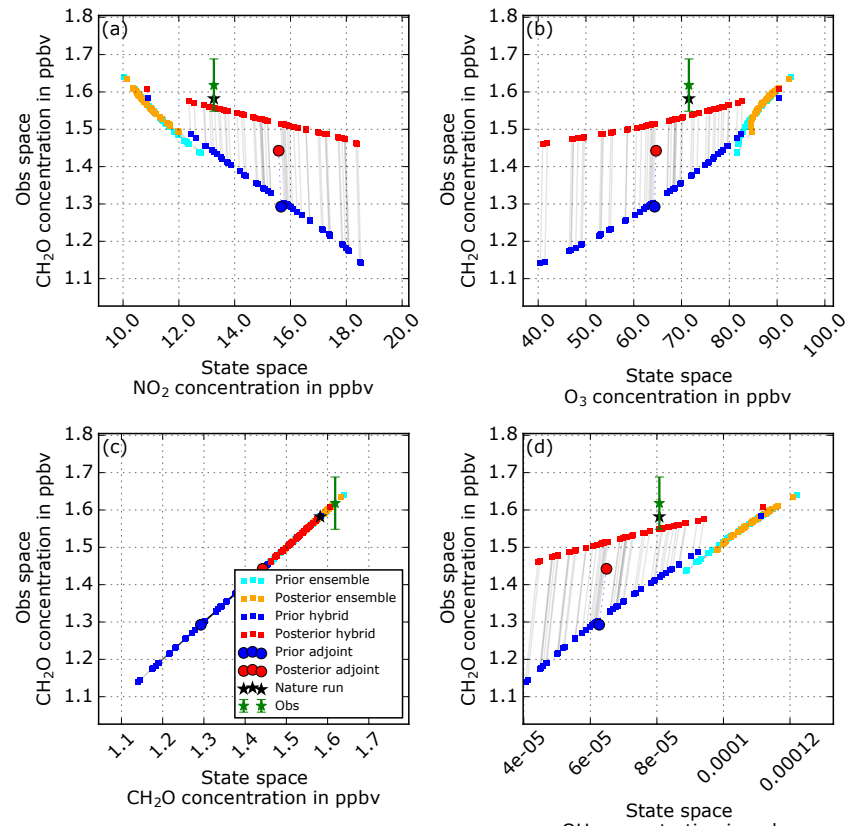

$\mathrm{OH}$ concentration in ppbv

Figure 8. Tracer-tracer or observation space to state space relationships during the ninth cycle of the $\mathrm{CH}_{2} \mathrm{O}$ assimilation without localization experiment.

concentration evolution) also differ. The production terms are stronger, leading to almost no loss net flux. The order of importance of the reactions in the budget is also different; $\mathrm{CH}_{2} \mathrm{O}+\mathrm{OH} \rightarrow \mathrm{CO}+\mathrm{HO}_{2}+\mathrm{H}_{2} \mathrm{O}$ is much more important, likely due to the increase in $\mathrm{OH}$ during the $\mathrm{AR}$ ensemble (see Sect. 3.2.2). Finally, the AR hybrid fluxes are similar to the CR fluxes with the same order of importance between reactions. Except for the carbon monoxide (CO) formation from photolysis, $\mathrm{CH}_{2} \mathrm{O} \rightarrow \mathrm{CO}+\mathrm{H}_{2}$ is now stronger and is responsible for the systematic decrease in $\mathrm{CH}_{2} \mathrm{O}$ in the AR adjoint and AR hybrid (see Sect. 3.2.2). This increased flux from this reaction explains the sawtooth behavior of $\mathrm{CH}_{2} \mathrm{O}$. When no other pathway of loss is possible, i.e., $\mathrm{OH}$ is not as strongly changed in AR adjoint and hybrid as in AR ensemble, this way of destroying $\mathrm{CH}_{2} \mathrm{O}$ is then increased.

We demonstrate here the usefulness of the flux tool to diagnose the effect of data assimilation on atmospheric chemistry in a detailed manner. The diagnostic of the flux tool can be used on any species that a chemical scheme contains for any kind of BOXMOX simulation.

\section{Summary and future options}

In this paper, we have presented a new suite of tools for box models, BEATBOX. The design of BEATBOX is based on an OSSE approach that can simultaneously integrate various chemical schemes to simulate a nature run, control runs, and assimilation runs. This framework includes the capabil-
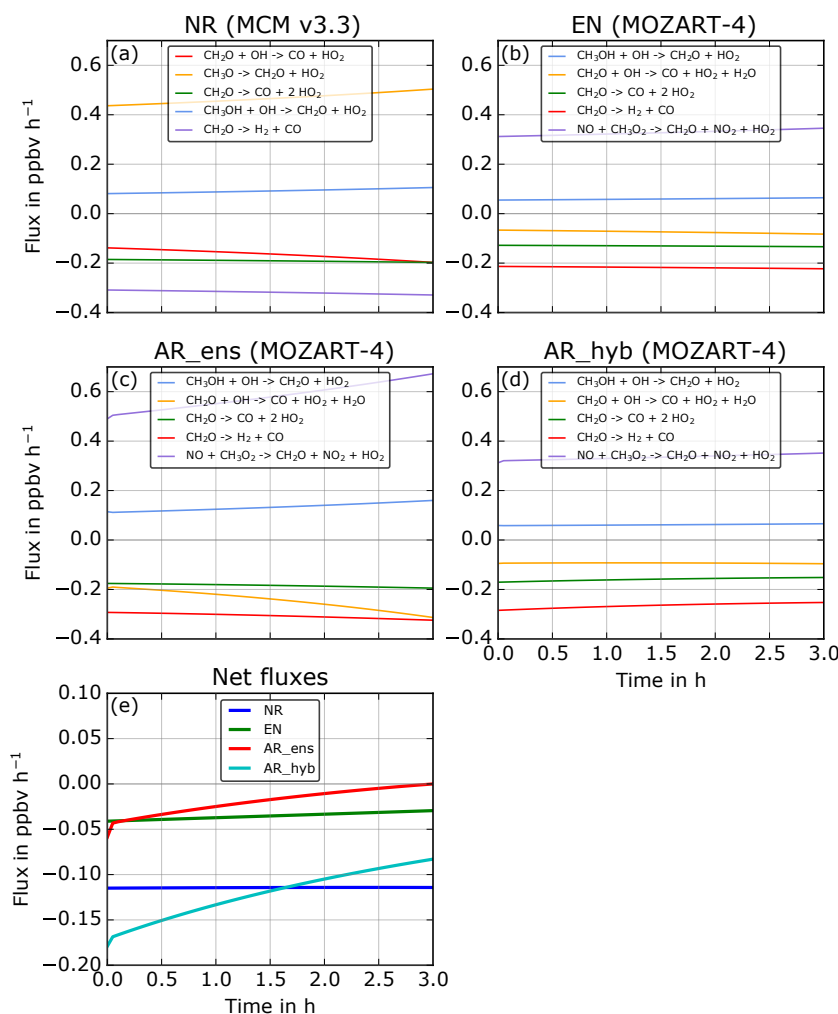

Figure 9. $\mathrm{CH}_{2} \mathrm{O}$ fluxes during the $3 \mathrm{~h}$ forecast of the ninth cycle of the $\mathrm{CH}_{2} \mathrm{O}$ assimilation with no localization. For the NR (a), one member of the CR (b), one member of the AR ensemble (c), and one member of the AR hybrid (d). Net fluxes for each run are shown in panel (e). Displayed are fluxes representative of $90 \%$ of the budget.

ity of running assimilation windows of different chemical schemes using a forecasting tool (BOXMOX) and an assimilation tool allowing for sensitivity analysis. BEATBOX provides ensemble and adjoint sensitivity analyses that can be combined or modified to explore new inverse or data assimilation methodologies. Additionally, a flux tool is also integrated into the framework to diagnose and assess in detail model run differences and ultimately use data assimilation to improve the model (chemical scheme) itself, not only the model outputs. The systematic and detailed assessment of the multivariate data assimilation problem indicates that BEATBOX can tackle important scientific hypotheses at a limited computational cost for future data assimilation configurations in large-scale 3-D models for atmospheric chemistry, but it is not limited to this. Any system of equations can be integrated over time in the current framework.

A typical case study of ozone photochemical production from $\mathrm{NO}_{x}$ is presented to showcase the capability of BEATBOX. Differences between the nature run and the control run are presented followed by a data assimilation experiment of synthetic $\mathrm{NO}_{2}$ and $\mathrm{CH}_{2} \mathrm{O}$ observations using adjoint and ensemble sensitivity analyses with different localization parameters. The case studies shown in this paper illustrate the need 
to understand in detail the effect of data assimilation in a complicated and nonlinear model as required by atmospheric chemistry. In these case studies, we showcased BEATBOX as a powerful and user-friendly tool for the following:

- understanding chemical mechanism differences and deficiencies,

- performing chemical sensitivity analysis using ensemble or adjoint methods,

- envisioning and designing new inverse and data assimilation methods for atmospheric chemistry to optimally constrain as much of the chemical state as possible,

- defining requirements for new chemical and data assimilation schemes and ultimately improving them, and

- educational purposes for data assimilation and atmospheric chemistry.

BEATBOX will continue to evolve according to user requirements. For example, emission inversion capability is currently being implemented. Setting any given observation time into the assimilation window will also be possible. Assimilating multiple observations in a given assimilation window will also be implemented using sequential and variational minimization techniques. Using real observations, i.e., from field campaigns, is also possible with minimal code modifications. Because of the user friendliness, flexibility, and open source nature of most of BOXMOX-BEATBOX, users could also contribute to model development and make it a broad atmospheric chemistry community tool.

Code and data availability. All code developments presented here are open source tools released under the GNU General Public License v3. BEATBOX consists of a number of Python packages:

- beatboxtestbed (the BEATBOX Background Error Analysis Testbed),

- boxmox (Python interface for the chemical box model BOXMOX),

- genbox (input data generator for boxmox),

- frappedata (FRAPPE campaign dataset for genbox),

- tuv (TUV data connector for genbox),

- chemspectranslator (translator to translate species between chemical mechanisms and observations), and

- icartt (reader-writer for ICARTT files).

The underlying chemical box model BOXMOX is a stand-alone $\mathrm{C}$ and Fortran executable and has to be installed before BEATBOX can be used.

Source code version 1.0 used in this paper is archived online at digital object identifier: 10.5281/zenodo.1118244 (Knote et al., 2017). Full documentation for BOXMOX and all Python packages, including examples on how to reproduce the case studies shown in this paper, can be found at https://boxmodeling.meteo.physik. uni-muenchen.de/documentation.
Author contributions. CK and JB contributed equally to the paper. CK designed the BOXMOX system, wrote the framework around BEATBOX, supervised ME, and contributed to writing the paper. JB came up with the idea for BEATBOX and coded most of the beatbox.py routine, co-supervised $\mathrm{ME}$, and wrote most of the paper. ME developed parts of the BEATBOX framework during his Masters thesis, prepared and analyzed the case studies, and contributed the flux tool.

Competing interests. The authors declare that they have no conflict of interest.

Acknowledgements. We thank the many scientists who contributed to the FRAPPE field campaign for providing useful data to initialize the model simulations. Frank Flocke and Rebecca Hornbrook (NCAR) are thanked for creating the Commerce City sample used in the case studies. We acknowledge Bill Carter (UC Riverside) for his work on the emission database. We acknowledge support from NASA KORUS-AQ grant NNX16AD96G.

Edited by: Tim Butler

Reviewed by: two anonymous referees

\section{References}

Anderson, J. L.: A local least squares framework for ensemble filtering, Mon. Weather Rev., 131, 634-642, 2003.

Anderson, J. L.: An adaptive covariance inflation error correction algorithm for ensemble filters, Tellus A, 59, 210-224, 2007.

Archibald, A. T., Jenkin, M. E., and Shallcross, D. E.: An isoprene mechanism intercomparison, Atmos. Environ., 44, 5356-5364, https://doi.org/10.1016/j.atmosenv.2009.09.016, 2010.

Arnold, C. P. and Dey, C. H.: Observing-systems simulation experiments: Past, present, and future, B. Amer. Meteorol. Soc., 67, 687-695, 1986.

Barré, J., Edwards, D., Worden, H., Silva, A. D., and Lahoz, W.: On the feasibility of monitoring carbon monoxide in the lower troposphere from a constellation of northern hemisphere geostationary satellites (part 1). Atmos. Environ., 113, 63-77, 2015.

Barré, J., Edwards, D., Worden, H., Arellano, A., Gaubert, B., Silva, A. D., Lahoz, W., and Anderson, J.: On the feasibility of monitoring carbon monoxide in the lower troposphere from a constellation of northern hemisphere geostationary satellites: Global scale assimilation experiments (part II), Atmos. Environ., 140, 188-201, 2016.

Claeyman, M., Attié, J.-L., Peuch, V.-H., El Amraoui, L., Lahoz, W. A., Josse, B., Joly, M., Barré, J., Ricaud, P., Massart, S., Piacentini, A., von Clarmann, T., Höpfner, M., Orphal, J., Flaud, J.-M., and Edwards, D. P.: A thermal infrared instrument onboard a geostationary platform for $\mathrm{CO}$ and $\mathrm{O}_{3}$ measurements in the lowermost troposphere: Observing System Simulation Experiments (OSSE), Atmos. Meas. Tech., 4, 1637-1661, https://doi.org/10.5194/amt-4-1637-2011, 2011.

Coates, J. and Butler, T. M.: A comparison of chemical mechanisms using tagged ozone production potential (TOPP) analysis, At- 
mos. Chem. Phys., 15, 8795-8808, https://doi.org/10.5194/acp15-8795-2015, 2015.

Edwards, D. P., Arellano Jr., A. F., and Deeter, M. N.: A satellite observation system simulation experiment for carbon monoxide in the lowermost troposphere, J. Geophys. Res., 114, D14304, https://doi.org/10.1029/2008JD011375, 2009.

Emmerson, K. M. and Evans, M. J.: Comparison of tropospheric gas-phase chemistry schemes for use within global models, Atmos. Chem. Phys., 9, 1831-1845, https://doi.org/10.5194/acp-91831-2009, 2009.

Emmons, L. K., Walters, S., Hess, P. G., Lamarque, J.-F., Pfister, G. G., Fillmore, D., Granier, C., Guenther, A., Kinnison, D., Laepple, T., Orlando, J., Tie, X., Tyndall, G., Wiedinmyer, C., Baughcum, S. L., and Kloster, S.: Description and evaluation of the Model for Ozone and Related chemical Tracers, version 4 (MOZART-4), Geosci. Model Dev., 3, 43-67, https://doi.org/10.5194/gmd-3-43-2010, 2010.

Fitzgerald, R.: Divergence of the Kalman filter, IEEE T. Automat. Contr., 16, 736-747, 1971.

Jenkin, M. E., Young, J. C., and Rickard, A. R.: The MCM v3.3.1 degradation scheme for isoprene, Atmos. Chem. Phys., 15, 11433-11459, https://doi.org/10.5194/acp-15-11433-2015, 2015.

Knote, C., Hodzic, A., Jimenez, J. L., Volkamer, R., Orlando, J. J., Baidar, S., Brioude, J., Fast, J., Gentner, D. R., Goldstein, A. H., Hayes, P. L., Knighton, W. B., Oetjen, H., Setyan, A., Stark, H., Thalman, R., Tyndall, G., Washenfelder, R., Waxman, E., and Zhang, Q.: Simulation of semi-explicit mechanisms of SOA formation from glyoxal in aerosol in a 3-D model, Atmos. Chem. Phys., 14, 6213-6239, https://doi.org/10.5194/acp14-6213-2014, 2014.

Knote, C., Tuccella, P., Curci, G., Emmons, L., Orlando, J. J., Madronich, S., Barò, R., Jimènez-Guerrero, P., Luecken, D., Hogrefe, C., Forkel, R., Werhahn, J., Hirtl, M., Pèrez, J. L., San Josè, R., Giordano, L., Brunner, D., Yahya, K., and Zhang, Y.: Influence of the choice of gas-phase mechanism on predictions of key gaseous pollutants during the AQMEII phase-2 intercomparison, Atmos. Environ., 115, 553-568, 2015.

Knote, C., Barré, J., and Eckl, M.: BEATBOX v1.0: Background Error Analysis Testbed with Box Models (Version 1.0.0), Zenodo, https://doi.org/10.5281/zenodo.1118244, 19 December 2017.

Kuo, Y.-H., Zou, X., and Huang, W.: The impact of global positioning system data on the prediction of an extratropical cyclone: an observing system simulation experiment, Dynam. Atmos. Oceans, 27, 439-470, 1998.

Lahoz, W., Khattatov, B., and Mènard, R. (Eds.): Data Assimilation: Making Sense of Observations, Springer Berlin Heidelberg, 718 pp., 2010.
Liu, C., Xiao, Q., and Wang, B.: An ensemble-based fourdimensional variational data assimilation scheme, part II: Observing system simulation experiments with advanced research WRF (ARW), Mon. Weather Rev., 137, 1687-1704, 2009.

Madronich, S. and Flocke, S.: Theoretical Estimation of Biologically Effective UV Radiation at the Earth's Surface, Springer, Berlin Heidelberg, 23-48, 1997.

Mazzuca, G. M., Ren, X., Loughner, C. P., Estes, M., Crawford, J. H., Pickering, K. E., Weinheimer, A. J., and Dickerson, R. R.: Ozone production and its sensitivity to $\mathrm{NO}_{x}$ and VOCs: results from the DISCOVER-AQ field experiment, Houston 2013, Atmos. Chem. Phys., 16, 14463-14474, https://doi.org/10.5194/acp-16-14463-2016, 2016.

Mitchell, H. L. and Houtekamer, P. L.: An adaptive ensemble Kalman filter, Mon. Weather Rev., 128, 416-433, 2000.

Nichols, N. K.: Mathematical Concepts of Data Assimilation, in: Data assimilation: making sense of observations, edited by: Lahoz, W., Khattatov, B. and Menard, R., Springer, 13-39, 2010.

Ott, E., Hunt, B., Szunyogh, I., Zimin, A., Kostelich, E., Corrazza, M., Kalnay, E., and Yorke, J.: A local ensemble Kalman filter for atmospheric data assimilation, Tellus A, 56, 415-428, 2004.

Sandu, A. and Sander, R.: Technical note: Simulating chemical systems in Fortran90 and Matlab with the Kinetic PreProcessor KPP-2.1, Atmos. Chem. Phys., 6, 187-195, https://doi.org/10.5194/acp-6-187-2006, 2006.

Sandu, A., Daescu D., and Carmichael G. R.: Direct and adjoint sensitivity analysis of chemical kinetic systems with KPP: Part I - theory and software tools, Atmos. Environ., 37, 5083-5096, https://doi.org/10.1016/j.atmosenv.2003.08.019, 2003.

Sandu, A., Daescu, D. N., Carmichael, G. R., and Chai, T.: Adjoint sensitivity analysis of regional air quality models, J. Comput. Phys., 204, 222-252, 2005.

van Leeuwen, P. J.: Nonlinear data assimilation in geosciences: an extremely efficient particle filter, Q. J. Roy. Meteor. Soc., 136, 1991-1999, https://doi.org/10.1002/qj.699, 2010.

Wang, X., Barker, D. M., Snyder, C., and Hamill, T. M.: A hybrid ETKF-3DVar data assimilation scheme for the WRF model, Part I: Observing system simulation experiment, Mon. Weather Rev., 136, 5116-5131, 2008

Wolfe, G. M., Marvin, M. R., Roberts, S. J., Travis, K. R., and Liao, J.: The Framework for 0-D Atmospheric Modeling (F0AM) v3.1, Geosci. Model Dev., 9, 3309-3319, https://doi.org/10.5194/gmd9-3309-2016, 2016. 